\title{
Mineral Luminescence Observed From Space
}

\author{
Philipp Khler ${ }^{1}$, Woodward W. Fischer ${ }^{1}$, George R. Rossman ${ }^{1}$, John P. \\ Grotzinger $^{1}$, Russell Doughty ${ }^{1}$, Yujie Wang ${ }^{1}$, Yi Yin ${ }^{1}$, Christian \\ Frankenberg ${ }^{1,2}$ \\ ${ }^{1}$ Division of Geological and Planetary Sciences, California Institute of Technology, Pasadena, CA, USA \\ ${ }^{2}$ Jet Propulsion Laboratory, California Institute of Technology, Pasadena, CA, USA
}

\section{Key Points:}

- First report of mineral luminescence observed from space

- Strongest signals originate from carbonate sedimentary rocks

- Emission appears to be spectrally flat in the NIR \& Lambertian

Corresponding author: Philipp Khler, Christian Frankenberg, pkoehler@caltech.edu, cfranken@caltech.edu

This article has been accepted for publication and ${ }^{-1}$ undergone full peer review but has not been through the copyediting, typesetting, pagination and proofreading process, which may lead to differences between this version and the Version of Record. Please cite this article as doi: 10.1029/2021GL095227.

This article is protected by copyright. All rights reserved. 


\begin{abstract}
Methods developed to explore the luminescent properties of the moon facilitated the development of techniques to infer terrestrial solar-induced chlorophyll fluorescence (SIF) from satellite instruments. While single SIF retrievals are inherently noisy, averaging many retrievals allows us to obtain highly accurate estimates. We analyzed several years of aggregated SIF data collected by the TROPOspheric Monitoring Instrument (TROPOMI) and the Orbiting Carbon Observatory-2 (OCO-2) over non-vegetated areas to explore the potential of SIF retrievals beyond the realm of photosynthesis. The fundamentally different retrievals at varying wavelengths in the near-infrared reveal that about $10 \%$ of all barren surfaces are weakly luminescent, while a few areas luminesce strongly — amounts comparable to SIF from vegetation. By means of lithological maps, we attributed the strongest luminescence signals to exposed carbonate sedimentary rocks. Besides a detailed evaluation of the signal properties, we discussed implications for SIF data sets and other remote sensing products.
\end{abstract}

\title{
Plain Language Summary
}

During the last decade, advancements in remote sensing techniques allowed us to infer solar-induced chlorophyll fluorescence, a faint electromagnetic signal emitted by vegetation, from space-borne measurements. Here, we explored the potential of such measurements to also observe mineral luminescence, a signal that is typically even weaker than fluorescence from vegetation, which explains why it has not been documented to be detectable from space. We identified luminescent regions by means of several years of vegetation fluorescence data derived from two satellites (OCO-2 and Sentinel-5P) over non-vegetated areas and analyzed the composition of luminescent surfaces. According to our analysis, about $10 \%$ of all barren areas on Earth are weakly luminescent, whereas some areas display surprisingly strong signals.

\section{Introduction}

Luminescence is the spontaneous emission of light by a substance that has absorbed and become excited by light, with the emission occurring at longer wavelengths associated with lower energy levels (known as Stokes shift). Studying this phenomenon has yielded a plethora of applications and insights across scientific fields such as biology, medicine, chemistry, and mineralogy (Gaft et al., 2015). While the vast majority of research in this regard deals with actively induced luminescence to identify the emitting molecules and study their mechanisms of energy transfer (fluorescence spectroscopy), luminescence can also be stimulated by solar irradiation and occurs naturally in rocks/minerals. The abundance and origins of mineral luminescence has traditionally been studied in laboratories by illuminating specimens with ultraviolet (UV) light and documenting the strength and wavelength of the resulting luminescence in the visible and infrared (Barnes, 1958; Gaft et al., 2015). Given the economic potential for prospecting of natural resources, passive remote sensing of mineral luminescence has been pursued since the 1960's (Hemphill \& Vickers, 1966). Despite early success detecting luminescent dyes from airborne platforms (Plascyk \& Gabriel, 1975), it became clear that solar-induced mineral luminescence (SML) was notoriously hard to detect with passive measurements because signal levels are typically extremely weak. Yet, advancements in spectrometers and the original Fraunhofer line-depth method developed to explore the luminescent properties of the moon (Kozyrev, 1956) enabled the remote sensing of solar-induced chlorophyll fluorescence (SIF) - an electromagnetic signal emitted by photosynthetically active vegetation (Porcar-Castell et al., 2014), which is about 100 times smaller than the reflected solar radiation. The emergence of global SIF data sets inferred from several Earth observing satellites (Mohammed et al., 2019) spurred an ever growing research interest due to its strong link to photosynthesis (Frankenberg et al., 2011; Guanter et al., 2012). 
From a mechanistic perspective, the photoelectric properties of certain mineral phases present at the Earth surface may provide inorganic photochemical analogs of photosynthesis (Tavakolian et al., 2019). In particular, the element manganese (Mn) has been identified to play a central role as its redox behavior allows oxygenic photosynthetic organisms (plants, algae, and Cyanobacteria) to access the high reduction-oxidation potentials needed to access water as electron donor (Fischer et al., 2015). This is done with the water-oxidizing complex $\left(\mathrm{Mn}_{4} \mathrm{CaO}_{5}\right)$ - a bioinorganic cluster of four $\mathrm{Mn}$ atoms and a Ca center bound by oxo-bridges, which can be found at the active site of photosystem II (Lingappa et al., 2019).

Despite typically faint signal levels, we might confuse SML with SIF in some areas of the Earth surface as satellite SIF retrievals are indifferent to the actual light source. There is some evidence that this phenomenon might be happening over barren regions (Joiner et al., 2012), but to date, this has not been further investigated, in part because SIF retrievals are typically noisy (high precision errors). On the other hand, SIF retrievals are thought to be very accurate (small biases) when many retrievals can be averaged.

We analyzed several years of SIF data collected by the TROPOspheric Monitoring Instrument (TROPOMI) and the Orbiting Carbon Observatory-2 (OCO-2) in areas devoid of vegetation with the goals to: 1) identify areas where SML can be observed, 2) examine what surface types are luminescent, and 3) assess properties of the emmitted signal. Being able to mask regions rich in SML is critical for improving SIF retrieval methods for studies aiming to estimate the carbon uptake by plants from global SIF data sets (MacBean et al., 2018; Smith et al., 2020). Moreover, it is known that any additional radiance can interfere with atmospheric trace gas retrievals (Lorente et al., 2021), thus acquiring knowledge about luminescent areas will help to identify and rectify potential biases.

\section{Methods \& Data}

\subsection{TROPOMI \& OCO-2 SIF}

TROPOMI (Veefkind et al., 2012) and OCO-2 (Crisp et al., 2017) are satellite instruments designed for atmospheric research, meaning that spatial detail is traded in exchange for the high spectral resolution needed to resolve narrow absorption lines from atmospheric trace gases. TROPOMI's single footprints measure $7 \mathrm{~km}$ along track $(5 \mathrm{~km}$ since August 2019 due to a reduced integration time) and $3.5-14 \mathrm{~km}$ across track (depending on the viewing angle), while the wide swath of $2600 \mathrm{~km}$ provides near-daily global surface coverage. OCO-2's swath of $11.3 \mathrm{~km}$ contains single footprints with a slightly better spatial resolution of $2.25 \mathrm{~km}$ along track and $1.3 \mathrm{~km}$ across track, which comes at the expense of providing a continuous spatial coverage - OCO-2 leaves large spatial gaps $(\approx$ 1.5 longitude) between successive orbits. Despite being designed for atmospheric research, the band setting of OCO-2 (band 1: 758-772 nm) and TROPOMI (band 5: 675-725 nm, band 6: $725-775 \mathrm{~nm}$ ) overlaps in parts with the wavelength range where SIF is emitted. Specifically, SIF occurs between $650-800 \mathrm{~nm}$, building a double peak feature with maximum values around $685 \mathrm{~nm}$ and $740 \mathrm{~nm}$. Retrieving the full SIF spectrum is challenging due to strong spectral variations in the surface and atmospheric reflectance as well as the atmospheric absorption, which is why current satellite based SIF retrievals evaluate only narrow wavelength ranges wherein reflectance and SIF can be assumed to be linear and wherein high atmospheric transmittance facilitates the modeling of the spectra.

Retrieval strategies exploited the reduced optical depth of solar Fraunhofer lines in the presence of SIF, yet fundamental differences between the retrieval algorithms of OCO-2 and TROPOMI exist. In the case of OCO-2, the $\mathrm{O}_{2}$-A band $(760-770 \mathrm{~nm})$ is measured with a spectral resolution of $0.04 \mathrm{~nm}$ to obtain additional information on cloud cover 
and surface pressure, which is necessary for the $\mathrm{CO}_{2}$ retrievals (O'Dell et al., 2018). However, atmospheric windows with sufficiently strong solar Fraunhofer lines are located around the $\mathrm{O}_{2}$-A band and micro-windows of just a few nanometers are used to infer SIF at $757 \mathrm{~nm}$ and $771 \mathrm{~nm}$ (Sun et al., 2018). The retrieval algorithm is physically-based, meaning that spectra are modeled using a reference data set of the solar irradiance, while instrumental effects (e.g., spectral broadening and shift) are state vector elements in the DOASlike SIF retrieval algorithm. In a post-processing step, a zero-level offset correction is performed (Sun et al., 2018) to account for subtle radiometric inconsistencies in OCO-2's spectra (Crisp et al., 2017).

TROPOMI's spectral resolution of $0.38 \mathrm{~nm}$ in the near-infrared (NIR) is inferior compared to OCO-2, even though a broader wavelength range is covered for the same purpose: to collect information about clouds and improve trace gas retrievals. To infer SIF from TROPOMI's spectra, a broader wavelength range - on the order of $15 \mathrm{~nm}-$ is necessary to obtain a sufficient signal-to-noise ratio. The SIF retrieval approach detailed in Köhler et al. (2018) can be characterized as statistical and data-driven. Specifically, a singular value decomposition of spectra over non-vegetated areas (training data set) provides spectral basis functions, which capture sensor specific characteristics and the depth of solar Fraunhofer lines in the absence of SIF. These spectral basis functions are then used in combination with a reference SIF shape to model the spectra between $743-758 \mathrm{~nm}$. The retrieval output consists of SIF at $740 \mathrm{~nm}$, corresponding to the second, longer wavelength, peak of the SIF emission spectrum.

Despite fundamental differences in instrument characteristics and SIF retrieval methods, Köhler et al. (2018) demonstrated that both data sets agree well over vegetated areas of the Earth surface when accounting for differences in evaluated wavelengths, acquisition times, and viewing-illumination geometries.

\subsection{Deriving Luminescent Surfaces}

To study SML emission from the Earth surface we began by excluding vegetated areas from the analysis using two additional data sets: 1) leaf area index (LAI; Yuan et al., 2011) provided on a 0.250 .25 grid and 2) International Geosphere and Biosphere Program (IGBP) land cover (Friedl et al., 2002) provided on a 0.050 .05 grid. The IGBP classification includes specifically a "Barren or Sparsely Vegetated" type, forming the basis for our analysis. To extend the analysis to the largest area possible, we also included areas classified as "Open/Closed Shrublands", "Woody Savannas", "Savannas", and "Grasslands" if the LAI was sufficiently low. Specifically, we generated a maximum LAI climatology and selected only regions out of the above mentioned IGBP classes where the maximum LAI throughout the season is below 0.5 , at which value the global distribution of TROPOMI SIF was still centered around zero. For comparison, the mean ( \pm SD) LAI for deserts has been reported to amount to $1.3( \pm 0.9)$ (Asner et al., 2003).

Single SIF retrievals display substantial uncertainties (on the order of $0.4 \mathrm{~mW} / \mathrm{m}^{2} / \mathrm{nm} / \mathrm{sr}$ ), which can complicate analyses over shorter time scales. However, thanks to the ever lengthening records of SIF, we can spatially aggregate (https://github.com/cfranken/gridding) several hundreds to thousands of retrievals and reduce the uncertainty of the mean by a factor of $\sqrt{N}$. The resulting long-term average (TROPOMI: 04/2018-07/2020, 0.05 x 0.05; OCO-2: $01 / 2015-12 / 2019,0.2 \times 0.2)$ is thought to be accurate, but note that small location-dependent biases may occur due to imperfections in the retrieval algorithms and/or post-processing steps. In fact, Turner et al. (2020) reported systematically negative TROPOMI $\mathrm{SIF}$ values in monthly averages over some barren regions in California, which are most likely related to bright surfaces associated with higher single retrieval uncertainties and issues with the selection of the training data. Considering current limitations, deriving SML areas from one data set alone might carry ambiguities due to idiosyncratic differences in the instrumental dataset and retrieval approach, but consensus signals observed 

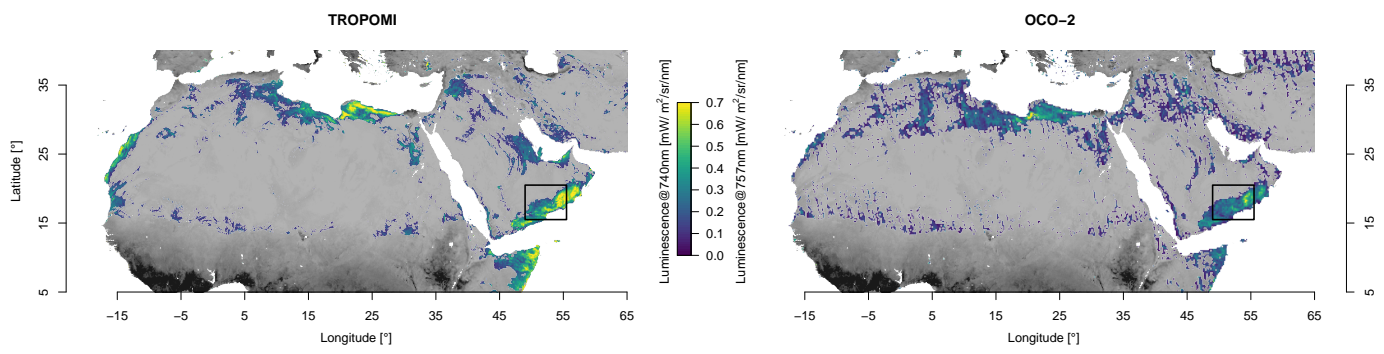

Figure 1. Maps of luminescent areas based on TROPOMI SIF at $740 \mathrm{~nm}$ averaged between $04 / 2018-07 / 2020$ on a $0.05 \times 0.05$ resolution and OCO-2 SIF at $757 \mathrm{~nm}$ averaged between $01 / 2015-12 / 2019$ on a $0.2 \times 0.2$ resolution. The black rectangle marks the area which is further investigated in Sect.3.3 and displayed in Fig. 3.

in two fundamentally different data sets provide a means to assess this and evaluate the robustness of the results. To facilitate this, we selected luminescent areas based on the coarser OCO-2 grid cells $(0.2 \times 0.2)$, where the long-term SIF averages of both TROPOMI and OCO-2 are larger than three times the standard error. After the initial analysis showed that SML tends to be spectrally flat in the relevant wavelength ranges (see Sect. 3.3), we added another filter criterium for the OCO-2 data: the difference between the two OCO-2 SIF averages (SIF@757-SIF@771nm) has to be smaller than $0.2 \mathrm{~mW} / \mathrm{m}^{2} / \mathrm{nm} / \mathrm{sr}$.

\subsection{Global Lithological Map Database (GLiM)}

The Global Lithological Map Database (GLiM) (Hartmann \& Moosdorf, 2012) describes the geochemical, mineralogical, and physical properties of rocks in unmatched detail. The database contains about 1.2 million spatial polygons of varying spatial resolutions and has been assembled from 92 regional geological maps. GLiM allowed us to explore SML surface types on three hierarchical levels: The first level contains 16 lithological main classes, while the additional two levels contain 12 and 14 sub-classes. We note that this approach does have some limitations due to occasional artificial geological boundaries placed at administrative borders and that the groundtruth evaluation of the GLiM results in only $40 \%$ accurate data points (64\% including similar lithologies). We considered these limitations to be of minor importance for the task of evaluating classifications at a global level.

\section{Results}

\subsection{Spatial Distribution}

We derived SML areas globally, however only North Africa and the Middle East are displayed in Fig. 1 because SML is most abundant in these areas of intense solar irradiation. In general, we observed that there is an excellent agreement in the spatial distribution of SML inferred from TROPOMI and OCO-2 independently; however, we proceeded to investigate only areas where both data sets agreed on the occurrence of SML.

Since the WGS84 coordinate system with lines of latitude parallel to the equator distorts the area of grid cells, we re-projected the 0.05 grid to an equal area projection (Eckert IV), which is also used by the GLiM database. In total, 124,075 SML grid cells (out of 18,600,270) were identified based on the criteria described in Sect. 2.2. This corresponded to $0.66 \%$ of the Earth's surface or $2.3 \%$ of the terrestrial Earth surface, which 

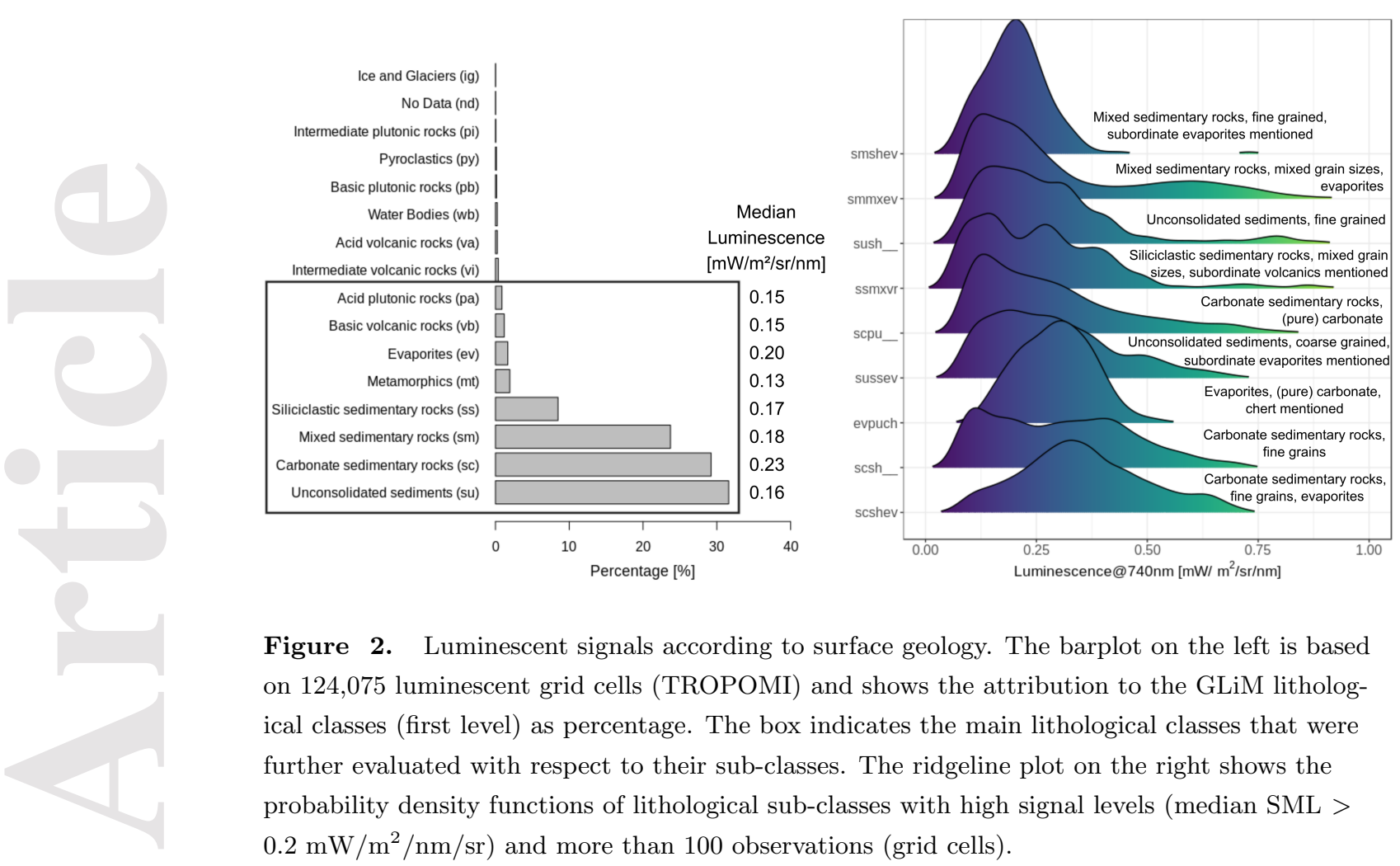

Figure 2. Luminescent signals according to surface geology. The barplot on the left is based on 124,075 luminescent grid cells (TROPOMI) and shows the attribution to the GLiM lithological classes (first level) as percentage. The box indicates the main lithological classes that were further evaluated with respect to their sub-classes. The ridgeline plot on the right shows the probability density functions of lithological sub-classes with high signal levels (median SML > $0.2 \mathrm{~mW} / \mathrm{m}^{2} / \mathrm{nm} / \mathrm{sr}$ ) and more than 100 observations (grid cells).

resulted in a surprisingly large fraction of barren surfaces $(\approx 10 \%)$ where SML may easily be confused with SIF from vegetation.

\subsection{Lithology}

To understand more about these potential SML signals, we selected only regions with a consensus on detected luminescent surfaces from OCO-2 and TROPOMI. The following analysis of lithological classes and sub-classes was based on TROPOMI SIF because the OCO-2 data had to be aggregated with coarser spatial resolution to mitigate the discontinuous spatial coverage.

This analysis indicated that the vast majority of SML areas could be broadly classified as 1) unconsolidated sediments, 2) carbonate sedimentary rocks, and 3) mixed sedimentary rocks (together $85 \%$, see Fig. 2). Relatively high median SML values were also observed for siliciclastic and evaporite sedimentary rocks (8.5/1.7\% of SML areas). In order to see whether or not the highly luminescent surfaces could be specified more precisely, we screened the lithological sub-classes of the first 8 main classes capturing $99 \%$ of all luminescent surfaces. This strategy yielded 152 sub-classes, so we screened these sub-classes further for high signal levels (median SML $>0.2 \mathrm{~mW} / \mathrm{m}^{2} / \mathrm{nm} / \mathrm{sr}$ ) and more than 100 observations (grid cells). The remaining 9 sub-classes are listed below (from higher to lower median values):

1. scshev: Carbonate sedimentary rocks (sc), fine grains (sh), evaporites (ev)

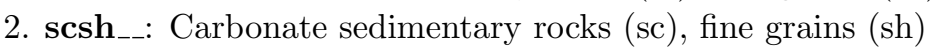

3. evpuch: Evaporites (ev), (pure) carbonate (pu), chert mentioned (ch)

4. sussev: Unconsolidated sediments (su), coarse grained (ss), subordinate evaporites mentioned (ev)

5. scpu $\mathbf{s}_{-.}$Carbonate sedimentary rocks (sc), (pure) carbonate (pu) 
6. ssmxvr: Siliciclastic sedimentary rocks (ss), mixed grain sizes (mx), subordinate volcanics mentioned (vr)

7. sush_-: Unconsolidated sediments (su), fine grained (sh)

8. smmxev: Mixed sedimentary rocks (sm), mixed grain sizes ( $\mathrm{mx}$ ), evaporites (ev)

9. smshev: Mixed sedimentary rocks ( $\mathrm{sm}$ ), fine grained (sh), subordinate evaporites mentioned (ev)

5 out of 9 sub-classes mention evaporites - a sedimentary rock type rich in soluble carbonate, sulfate, and chloride salts formed by evaporation - and 4 sub-classes include carbonate explicitly. Despite evaporites contributed only 1.7\% to all SML areas (main classification), the high median SML value and the frequency of occurrence in the high signal sub-classes suggested that evaporite-bearing units are common emitters. However, a closer inspection of the Miocene evaporites of north Africa and halite glaciers (salt domes) of the Zagros mountains revealed that neither gypsum or halite evaporites were correlated with the SML signal. Considering a prevalent spatial heterogeneity and mutual occurrence, we hypothesized that the SML emission is more likely to originate from carbonate sedimentary rocks. Siliciclastic sedimentary rocks were most abundant in $8.5 \%$ of the identified SML areas and also represented in the high signal sub-classes, which suggested that this rock type should be considered as a potential SML emitter as well.

The probability density functions of the 9 strongest luminescent surfaces in Fig. 2 were characterized by fat tails towards higher signal levels and median SML values of about $0.25 \mathrm{~mW} / \mathrm{m}^{2} / \mathrm{nm} / \mathrm{sr}$. The weak magnitude illustrated why several years of data were needed to detect SML areas robustly, while a small number of grid cells displays surprisingly high values.

\subsection{Characterizing SML based on Oman/Yemen}

The Oman/Yemen region exhibited the highest SML values on Earth, and consequently we selected this region for a more detailed analysis.

Fig. 3 shows a high degree of similarity between TROPOMI and OCO-2 results, with clear boundaries between apparently inert sand dunes and consolidated Tertiary carbonates (mostly limestone). Carbonates often have $\mathrm{Mn}^{2+}$ substitutions at low concentrations, which are well known to act as activator causing luminescence under UV light (El Ali et al., 1993). In contrast, sand dunes are typically composed of minerals phases like quartz $\left(\mathrm{SiO}_{2}\right)$, and or silicates with $\mathrm{Fe}^{2+}$ (e.g. olivines, pyroxenes), which tend not to luminesce. Intense solar radiation and $\mathrm{Mn}^{2+}$ impurities in carbonate rich rocks may consequently explain why SML can be observed in this region, yet remarkably the power is much higher than in other barren areas. Some areas in Fig. 3 displayed an average emission of about $0.8 \mathrm{~mW} / \mathrm{m}^{2} / \mathrm{nm} / \mathrm{sr}$, which corresponds to the values seen in the fat tails in the globally aggregated probability distributions in Fig. 2. This signal level was comparable to many vegetated areas around the globe and highlighted the importance of being able to accurately deconvolve SIF and SML signals in barren areas of the Earth surface. Notably, the nearly identical magnitudes between TROPOMI $(740 \mathrm{~nm})$ and OCO$2(757 \mathrm{~nm}$ and $771 \mathrm{~nm})$ suggested that the signal is relatively flat in the NIR.

We evaluated directional properties of SML/SIF and reflected radiance levels over non-luminescent, SML, and vegetated areas as a function of the observational phase anglethe angle between the directions to the sun and detector as seen from the surface- based on single sounding (ungridded) TROPOMI data in Fig. 4. The reflected radiance levels (right column of Fig. 4) were sampled over surfaces with slightly different brightness levels, which is reflected in the error bars representing the standard deviation per phase angle bin. In contrast to the profound effects of the viewing-illumination geometry on reflected radiances and SIF over vegetation, we expected that SML would be Lambertian because the emission originates in the uppermost surface layer without being scat- 

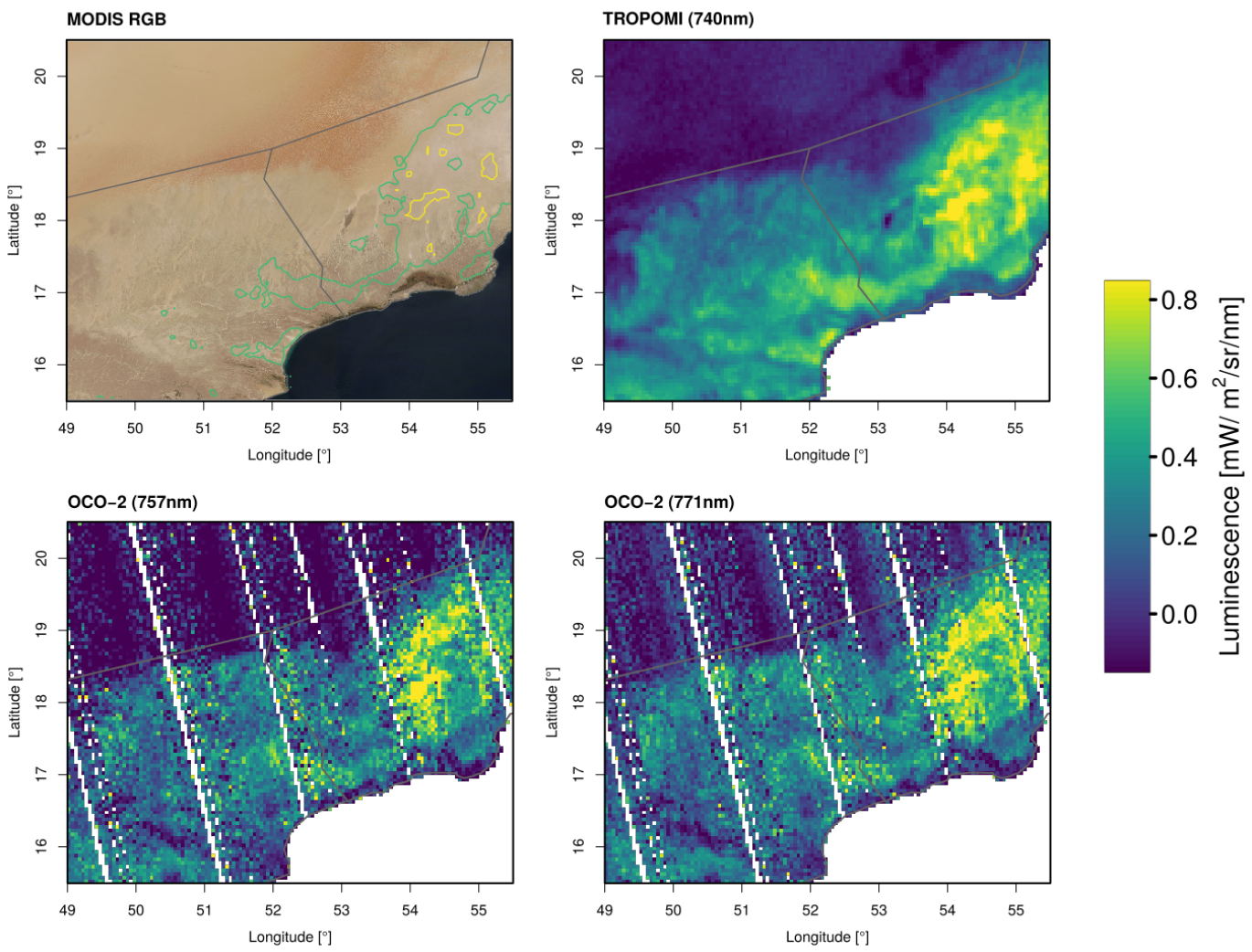

Figure 3. Luminescent signals from Oman and Yemen are the largest on Earth. The MODIS RGB image $(07 / 11 / 2018)$ of the study area $(49 \mathrm{E}-55.5 \mathrm{E}, 15.5 \mathrm{~N}-20.5 \mathrm{~N})$ shown together with SML contour lines of high intensity (above $0.54 / 0.84 \mathrm{~mW} / \mathrm{m}^{2} / \mathrm{nm} / \mathrm{sr}$, based on TROPOMI) on the top left. The other maps display TROPOMI and OCO-2 averages (04/2018 - 07/2020) at $740 \mathrm{~nm}, 757 \mathrm{~nm}$, and $771 \mathrm{~nm}$.

tered. Based on the phase angle, the middle panel of Fig. 4 illustrates that this assumption is fairly accurate. Similarly, the non-luminescent desert should show no preferential directionality, which was supported by the data in the first panel of Fig. 4. It may be noted that fluctuations around the expected zero could be explained by the uncertainty as the actual probability density (embedded barplot) matched the expected distribution (red line) remarkably well. For comparison, the bottom panel of Fig. 4 illustrated the typical anisotropic SIF emission characteristics from vegetation with the hotspot at 0 phase angle, where the fully illuminated parts of the canopy without shadowing effects were observed. Vegetation was less reflective in the concerned wavelength range $(743 \mathrm{~nm}$ $-758.5 \mathrm{~nm}$ ) compared to the bright desert and displayed a stronger hotspot effect without a secondary peak. We found that the reflected radiance levels (right column of Fig. 4) of the non-luminescent and luminescent desert were very similar, showing a directionality with a preference for back-scattering into the hotspot direction and a second local maximum at $\approx-60$ phase angle. If SML signals were due to reflectance properties, a similar phase angle dependency (directionality) compared to the reflected radiance levels could be observed, but this was not the case. The validity of our retrievals was further corroborated by the SML signal size, which was independent of the brightness level as the non-luminescent areas were on average slightly brighter than the SML areas. 

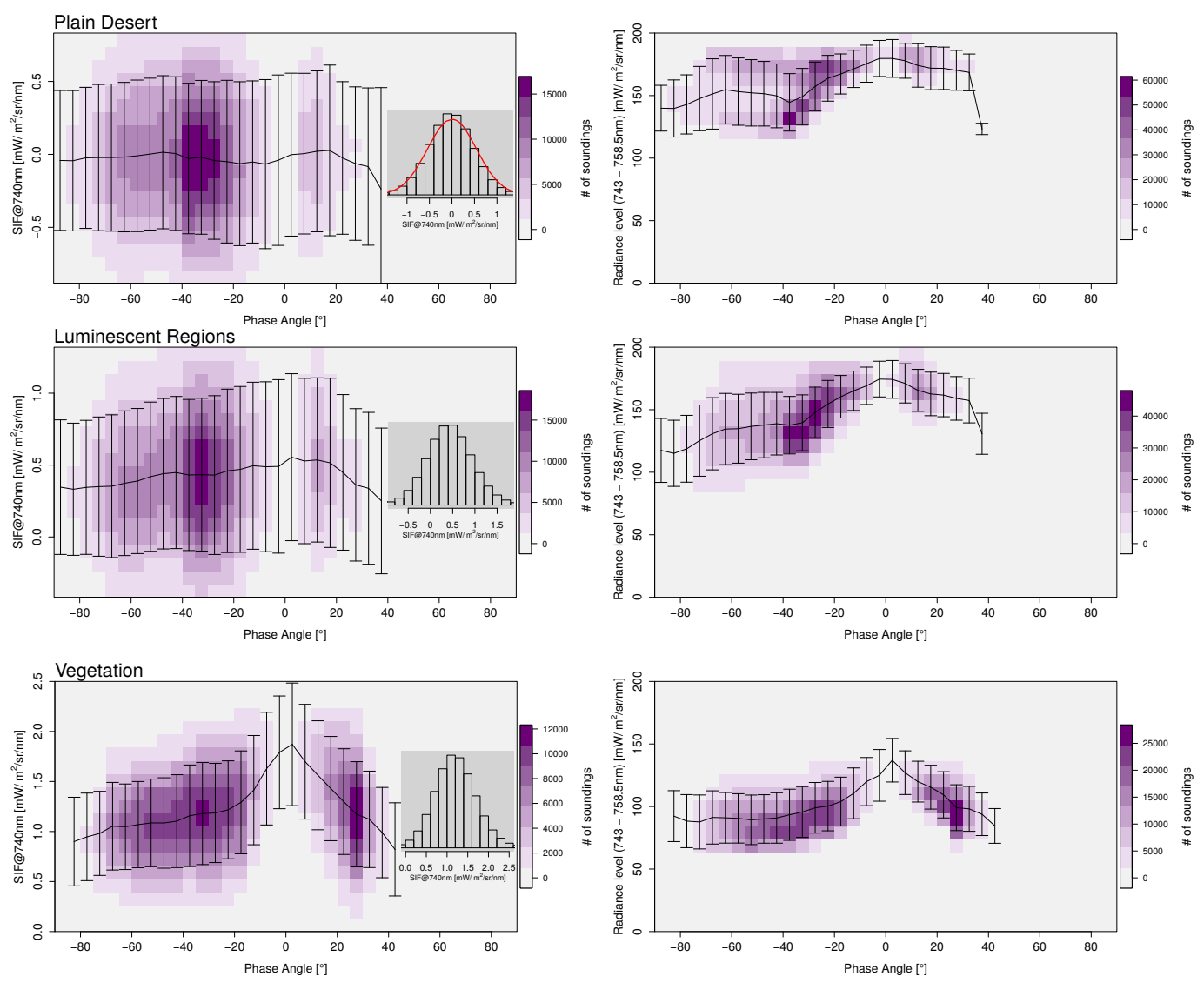

Figure 4. Displayed are SML, SIF, and reflected radiance levels (TROPOMI single retrievals) as a function of the phase angle for three different surface types: non-/lumincescent desert and vegetation. While the desert data is based on the Oman/Yemen region shown in Fig. 3, the vegetation data has been sampled from the inner Maiko National Park in the Democratic Republic of the Congo (tropical forest, $2 \mathrm{~S}-2 \mathrm{~N}, 19 \mathrm{E}-23 \mathrm{E}$ ) for the available data between $11 / 23 / 2017$ and $04 / 30 / 2020$. The embedded barplots in the left column show the probability distribution of SML/SIF, where the red line in the first plot represents the predicted uncertainty based on the mean uncertainty of the concerned retrievals $\left(0.46 \mathrm{~mW} / \mathrm{m}^{2} / \mathrm{nm} / \mathrm{sr}\right)$. The error bars represent the standard deviation per phase angle bin. 
We also investigated whether or not temporal patterns over SML areas exist, but observed no seasonality in the SML signal to date.

\section{Discussion}

We observed consistent spatial patterns and magnitudes between retrievals from TROPOMI and OCO-2 in barren areas of the Earth surface that provided evidence for the detectability of SML from space. One caveat with our analysis consisted of the challenge to exclude vegetation entirely for satellite observations with footprint sizes of a few kilometers, which could potentially result in some falsely identified SML areas.

Current SIF retrievals leverage the assumption that one can use observations of nonfluorescent barren surfaces to train data-driven retrievals (TROPOMI, GOME, SCIAMACHY, GOME-2) or perform zero-level offset corrections (GOSAT, OCO-2/3, TANSAT). We found that this assumption is violated in some cases due to areas with SML, which could potentially introduce a negative bias into global SIF data sets.

Even though Turner et al. (2020) found small negative biases in monthly averaged TROPOMI data, it should be mentioned that the magnitude of those biases is still consistent with the uncertainty estimates. So far, we have not observed consistently negative/positive biases in the TROPOMI or the OCO-2 data, which would justify a uniform global correction. However, based on these observations we recommend the exclusion of such luminescent surfaces when selecting training data and performing zero-level offset corrections in future iterations of SIF retrieval algorithms to mitigate this potential bias source. Luminescent areas should also be excluded (e.g. by masking) in analyses aiming to estimate the gross primary production (GPP) through photosynthesis from SIF data sets as the additional signal may result in a high bias of GPP estimates.

Future studies may explore if dedicated retrievals at distinct wavelengths carry more information about the compositions and material properties of minerals exposed at the Earth surface. Given the rather low 'ground-truth' accuracy of the GLiM database, such studies might profitably target areas of high SML intensity and well documented surface properties. We hypothesized that $\mathrm{Mn}^{2+}$ impurities may cause SML in the Oman/Yemen region, but it should be mentioned that lab measurements by Aguilar \& Osendi (1982) have shown that the emission peak of $\mathrm{Mn}^{2+}$ impurities in $\mathrm{CaCO}_{3}$ - the main compound of carbonate sedimentary rocks such as limestone occurs at $610 \mathrm{~nm}$. Other potential activators capable of generating luminescence in the concerned wavelength range (740$771 \mathrm{~nm})$ are $\mathrm{Fe}^{3+}$ and $\mathrm{Cr}^{3+}$ Gaft et al. (2015). The answer to the question about the actual activator is further complicated by trace contamination with other ions, which may act as sensitizers and co-activators, reflecting the rich photochemistry occurring with mineral phases at the Earth surface. Interestingly this is a similar type of photochemistry that takes place in the water-oxidizing complexes of photosystem II held by all oxygenic photosynthetic organisms.

A more detailed analysis of SML emission wavelengths and strengths may not only enable observations of spectral profiles, but also help to identify sources of errors for trace gas retrievals, which rely on the $\mathrm{O}_{2}$ A-band to account for atmospheric effects due to scattering in optically thin cirrus clouds and/or aerosol layers as well as surface pressure.

\section{Conclusions}

We analyzed several years of aggregated TROPOMI and OCO-2 SIF data over nonvegetated areas to identify regions of the Earth surface where SML occurs. We found that about $10 \%$ of barren surfaces are weakly luminescent, however there are several arid regions wherein SML is on the same order of magnitude as SIF signals from vegetation elsewhere. We attributed the strongest SML signals to landscapes with carbonate-rich 
sedimentary bedrock in areas of intense solar irradiation. A closer inspection of the exceptionally strong luminescent Oman/Yemen region revealed that SML tends to be spectrally flat in the $743-771 \mathrm{~nm}$ wavelength range. In contrast to the reflected radiance and SIF, the SML emission can be characterized as Lambertian. Knowledge about affected areas is valuable for understanding Earth surface properties and processes, but it is also beneficial for improving future SIF retrieval algorithms and obtaining more accurate SIF data.

\section{Acknowledgments}

This work was funded by the EARTH SCIENCE U.S. PARTICIPATING INVESTIGATOR (Grant: NNX15AH95G). Sentinel-5 Precursor is a European Space Agency (ESA) mission implemented on behalf of the European Commission (EC). The TROPOMI payload is a joint development by ESA and the Netherlands Space Office (NSO). The Sentinel5 Precursor ground segment development has been funded by ESA and with national contributions from the Netherlands, Germany, Belgium, and UK. The TROPOMI SIF data can be accessed through https://doi.org/10.22002/D1.1347 and luminescent areas through https://doi.org/10.22002/D1.2033.

\section{References}

Aguilar, G., \& Osendi, M. (1982). Fluorescence of mn2+ in caco3. Journal of Luminescence, $27(4), 365-375$.

Asner, G. P., Scurlock, J. M., \& A. Hicke, J. (2003). Global synthesis of leaf area index observations: implications for ecological and remote sensing studies. Global Ecology and Biogeography, 12(3), 191-205.

Barnes, D. F. (1958). Infrared luminescence of minerals (No. 1052). US Government Printing Office.

Crisp, D., Pollock, H. R., Rosenberg, R., Chapsky, L., Lee, R. A., Oyafuso, F. A., ... others (2017). The on-orbit performance of the orbiting carbon observatory2 (oco-2) instrument and its radiometrically calibrated products. Atmospheric Measurement Techniques, 10(1), 59-81.

El Ali, A., Barbin, V., Calas, G., Cervelle, B., Ramseyer, K., \& Bouroulec, J. (1993). $\mathrm{Mn} 2+$-activated luminescence in dolomite, calcite and magnesite: quantitative determination of manganese and site distribution by epr and cl spectroscopy. Chemical Geology, 104 (1-4), 189-202.

Fischer, W. W., Hemp, J., \& Johnson, J. E. (2015). Manganese and the evolution of photosynthesis. Origins of Life and Evolution of Biospheres, 45 (3), 351-357.

Frankenberg, C., Fisher, J. B., Worden, J., Badgley, G., Saatchi, S. S., Lee, J.-E., ... others (2011). New global observations of the terrestrial carbon cycle from gosat: Patterns of plant fluorescence with gross primary productivity. Geophysical Research Letters, 38(17).

Friedl, M. A., McIver, D. K., Hodges, J. C., Zhang, X. Y., Muchoney, D., Strahler, A. H., ... others (2002). Global land cover mapping from modis: algorithms and early results. Remote sensing of Environment, 83(1-2), 287-302.

Gaft, M., Reisfeld, R., \& Panczer, G. (2015). Modern luminescence spectroscopy of minerals and materials. Springer.

Guanter, L., Frankenberg, C., Dudhia, A., Lewis, P. E., Gómez-Dans, J., Kuze, A., ... Grainger, R. G. (2012). Retrieval and global assessment of terrestrial chlorophyll fluorescence from gosat space measurements. Remote Sensing of Environment, 121, 236-251.

Hartmann, J., \& Moosdorf, N. (2012). The new global lithological map database GLiM: A representation of rock properties at the Earth surface. Geochemistry, Geophysics, Geosystems, 13(12). 
Hemphill, W., \& Vickers, R. (1966). Geological studies of the earth and planetary surfaces of ultraviolet absorption and simulated luminescence.

Joiner, J., Yoshida, Y., Vasilkov, A., Middleton, E., Campbell, P., Kuze, A., \& Corp, L. (2012). Filling-in of near-infrared solar lines by terrestrial fluorescence and other geophysical effects: Simulations and space-based observations from SCIAMACHY and GOSAT. Atmospheric Measurement Techniques, 5(4), 809-829.

Köhler, P., Frankenberg, C., Magney, T. S., Guanter, L., Joiner, J., \& Landgraf, J. (2018). Global retrievals of solar-induced chlorophyll fluorescence with TROPOMI: First results and intersensor comparison to OCO-2. Geophysical Research Letters, 45(19), 10-456.

Kozyrev, N. (1956). Luminescence of the lunar surface and intensity of corpuscular radiation of the sun. Proceedings of the Crimean Astron. Observatory, 16, 148158.

Lingappa, U. F., Monteverde, D. R., Magyar, J. S., Valentine, J. S., \& Fischer, W. W. (2019). How manganese empowered life with dioxygen (and vice versa). Free Radical Biology and Medicine, 140, 113-125.

Lorente, A., Borsdorff, T., Butz, A., Hasekamp, O., Schneider, A., Wu, L., ... others (2021). Methane retrieved from tropomi: improvement of the data product and validation of the first 2 years of measurements. Atmospheric Measurement Techniques, $14(1), 665-684$.

MacBean, N., Maignan, F., Bacour, C., Lewis, P., Peylin, P., Guanter, L., ... Disney, M. (2018). Strong constraint on modelled global carbon uptake using solarinduced chlorophyll fluorescence data. Scientific reports, 8(1), 1-12.

Mohammed, G. H., Colombo, R., Middleton, E. M., Rascher, U., van der Tol, C., Nedbal, L., ... others (2019). Remote sensing of solar-induced chlorophyll fluorescence (sif) in vegetation: 50 years of progress. Remote sensing of environment, 231, 111177.

O'Dell, C. W., Eldering, A., Wennberg, P. O., Crisp, D., Gunson, M. R., Fisher, B., ... others (2018). Improved retrievals of carbon dioxide from orbiting carbon observatory-2 with the version 8 acos algorithm. Atmospheric Measurement Techniques, 11(12), 6539-6576.

Plascyk, J. A., \& Gabriel, F. C. (1975). The fraunhofer line discriminator mkii-an airborne instrument for precise and standardized ecological luminescence measurement. IEEE Transactions on Instrumentation and measurement, 24(4), 306-313.

Porcar-Castell, A., Tyystjärvi, E., Atherton, J., Van der Tol, C., Flexas, J., Pfündel, E. E., .. Berry, J. A. (2014). Linking chlorophyll a fluorescence to photosynthesis for remote sensing applications: mechanisms and challenges. Journal of experimental botany, 65(15), 4065-4095.

Smith, W. K., Fox, A. M., MacBean, N., Moore, D. J., \& Parazoo, N. C. Constraining estimates of terrestrial carbon uptake: New opportunities using long-term satellite observations and data assimilation. New Phytologist, 225(1), $105-112$.

Sun, Y., Frankenberg, C., Jung, M., Joiner, J., Guanter, L., Köhler, P., \& Magney, T. (2018). Overview of solar-induced chlorophyll fluorescence (sif) from the orbiting carbon observatory-2: Retrieval, cross-mission comparison, and global monitoring for gpp. Remote Sensing of Environment, 209, 808-823.

Tavakolian, M., Salimi, P., Zand, Z., \& Najafpour, M. M. (2019). Chapter three water oxidation by manganese oxides. In R. van Eldik \& C. D. Hubbard (Eds.), Water oxidation catalysts (Vol. 74, p. 115-150). Academic Press. Retrieved from https://www.sciencedirect. com/science/article/pii/S0898883819300200 doi: https://doi.org/10.1016/bs.adioch.2019.03.003

Turner, A. J., Köhler, P., Magney, T. S., Frankenberg, C., Fung, I., \& Cohen, R. C. (2020). A double peak in the seasonality of california's photosynthesis as observed from space. Biogeosciences, 17(2), 405-422. 
Veefkind, J., Aben, I., McMullan, K., Förster, H., De Vries, J., Otter, G., .. others (2012). Tropomi on the esa sentinel-5 precursor: A gmes mission for global observations of the atmospheric composition for climate, air quality and ozone layer applications. Remote sensing of environment, 120, 70-83.

Yuan, H., Dai, Y., Xiao, Z., Ji, D., \& Shangguan, W. (2011). Reprocessing the modis leaf area index products for land surface and climate modelling. Remote Sensing of Environment, 115(5), 1171-1187. 